\title{
Diagnostic algorithm and therapeutic options in chronic heart failure: updated review of clinical practice guidelines
}

\author{
Grzegorz Gajos \\ Department of Coronary Artery Diseases, Institute of Cardiology, Collegium Medicum, Jagiellonian University, John Paul II Hospital, Kraków, Poland
}

\section{KEY WORDS}

chronic heart failure, diagnostic techniques, practice guidelines, therapy
Correspondence to: Grzegorz Gajos, MD, PhD,

Krakowski Szpital Specjalistyczny im. Jana Pawla II, Klinika Choroby Wieńcowej Instytutu Kardiologii CM UJ, ul. Prądnicka 80, 31-202 Kraków, Poland, phone: + 48-12-633-67-44, fax: +48-12-633-67-44, e-mail: ggajos@szpitaljp2.krakow.pl Received: March 2, 2008. Revision accepted: April 22, 2008. Conflict of interest: none declared. Pol Arch Med Wewn. 2008; 118 (9): 489-500

Translated by Iwona Rywczak, MD, PhD

Copyright by Medycyna Praktyczna, Kraków 2008

\begin{abstract}
The development of diagnostic and therapeutic algorithms is becoming more and more popular in medicine. Their advantage is simplicity which enables wide use in everyday medical practice. The algorithms may have limitations mainly due to simplifications, which not always appear to be helpful in solving complex clinical problems of an individual patient. To serve as a "signpost" in clinical practice, the algorithm should be worked out strictly according to the appropriately interpreted data obtained from reliable, current and published medical evidence. This aim is achieved by clinical practice guidelines, being developed by scientific societies. The present paper deals with diagnostic and therapeutic algorithms in chronic heart failure based on the current clinical practice guidelines. The current guidelines by the European Society of Cardiology have been compared with similar documents issued by the American College of Cardiology/American Heart Association, the Heart Failure Society of America and the Canadian Cardiovascular Society. Similarities and divergences between the guidelines of different societies in respect of treatment for chronic heart failure have been discussed. The detailed description of their role in the treatment has been provided for: non-pharmacological measures (guidelines for lifestyle), drug therapy (angiotensin-converting enzyme inhibitors, $\beta$-adrenolytics, aldosterone receptor antagonists, angiotensin receptor blockers, cardiac glycosides and diuretics), implantation of cardioverter-defibrillators and(or) resynchronization device, mechanical support and surgical treatment. The paper also focuses on diagnostic problems and treatment modalities for heart failure with a preserved systolic function of the left ventricle.
\end{abstract}

Working out diagnostic and therapeutic algorithms in medicine is becoming essential on account of that it increases knowledge originating from a considerable number of published reports on clinical trials. The simplicity of such algorithms enables their wide application in everyday medical practice. The algorithms may have limitations mainly due to simplifications, which not always turn out to be useful in solving complex clinical problems of an individual patient. To serve as "signpost" in clinical practice, the scheme ought to be worked out strictly according to the appropriately interpreted data originating from reliable, current and published medical information. This aim is accomplished by clinical practice guidelines, being worked out by scientific societies. It should be emphasized that the algorithms presented in such guidelines need to be one of the components taken into account during clinical decisions, and their application should consider individual assessment of a clinical situation. It is also worth mentioning that the guidelines are prepared mainly based on data coming from large, randomized controlled trials. The nature of those trials, and, in particular, a selective choice of patients, limits their approach towards some groups of patients, particularly the elderly, patients with serious concomitant diseases and multiple risk factors, and females. These are the patients, who are often not enrolled in randomized clinical trials. Those limitations resulting from interpretation of clinical trials should be 
TABLE 1 Heart failure classification according to NYHA

\begin{tabular}{cc}
\hline Class I & $\begin{array}{c}\text { No limitation of physical activity. Ordinary physical activity does not } \\
\text { cause undue fatigue, palpitation, or dyspnea (shortness of breath). }\end{array}$ \\
\hline Class II & $\begin{array}{c}\text { Slight limitation of physical activity. Comfortable at rest, but ordinary } \\
\text { physical activity results in fatigue, palpitation, or dyspnea. }\end{array}$ \\
\hline Class III & $\begin{array}{c}\text { Marked limitation of physical activity. Comfortable at rest, but less } \\
\text { than ordinary activity causes fatigue, palpitation, or dyspnea. }\end{array}$ \\
\hline Class IV & $\begin{array}{c}\text { Unable to carry out any physical activity without discomfort. } \\
\text { Symptoms of cardiac insufficiency at rest. If any physical activity } \\
\text { is undertaken, discomfort is increased. }\end{array}$ \\
\hline
\end{tabular}

particularly considered in the population of Polish patients with heart failure, especially as they may overlap with limited following the doctor's recommendations. Individual conditions of the patients, including their financial situation and high prices they pay for complex pharmacotherapy, influence the way Polish patients follow the rules of long-time therapy in heart failure. Attention should be paid to the fact that the effectiveness of treatment in heart failure is associated with not only of properly applied therapeutic strategies, but also a patient's appropriate behavior and cooperation.

Within the field of treatment for chronic heart failure ( $\mathrm{CHF}$ ) there are four main sets of guidelines developed by:

1) European Society of Cardiology (ESC) of $2005^{1}$

2) American College of Cardiology/American Heart Association (ACC/AHA) of $2005^{2}$

3) Heart Failure Society Of America (HFSA) of $2006^{3}$

4) Canadian Cardiovascular Society (CCS) of 2006 (updated in 2007). ${ }^{4}$

As the Polish Cardiac Society recommended implementation of the European guidelines in Poland, the diagnostic and therapeutic algorithms presented below are compliant with those guidelines. The existing differences between the mentioned recommendations are included in the commentaries (titled "Comparison between the guidelines...").

Guidelines for scientific societies have been divided into recommendation classes, considering the level of scientific evidence supporting the recommendations.

\section{Recommendation classes}

1) Class I - there is scientific evidence and(or) general agreement that the analyzed diagnostic procedure/method of treatment is beneficial, useful and effective.

2) Class II - data from studies are ambiguous and(or) there exist divergent opinions concerning the usefulness/effectiveness of a given form of therapy.

a) Class IIa - evidence/opinions confirming the usefulness/effectiveness of a method predominate.

European Society of Cardiology advise not to apply procedures included in class III
3) Class III* - there is scientific evidence or general agreement that a method of treatment is useless/ineffective, and sometimes may be harmful.

\section{Gradation of scientific evidence}

1) Level A - data derived from multiple randomized clinical trials or from meta-analyses.

2) Level B - data derived from a single randomized clinical trial or several large non-randomized trials.

3) Level $C$ - concurrent opinion of experts and(or) results of small studies; data from retrospective studies and registers.

Diagnostic algorithms in CHF Diagnosis of CHF is first of all clinical made based on subjective and objective symptoms. Properly selected tests help confirm the diagnosis, although it should be emphasized that a single, specific test enabling heart failure diagnosis is not available.

A definition of heart failure, according to the guidelines of the ESC, underlines the necessity of existence of: ${ }^{4}$

1) symptoms of heart failure (at rest or with exertion) and

2) objective (preferably echocardiographic) indications of cardiac dysfunction (systolic and[or] diastolic) at rest

3) and, in case of diagnostic doubts, positive response to treatment directed towards heart failure (HF).

Criteria 1 and 2 have to be fulfilled at all events.

Based on such a definition, an appropriate scheme for proper diagnosis of heart failure can be produced (FIGURE 1). Interpretation of the symptoms mentioned above in clinical practice may be difficult and it requires taking the patient's age, sex, obesity and concomitant diseases into consideration. It is also well known that the relationship between in symptoms of heart failure and the degree of cardiac dysfunction is weak, and long-term prognosis depends mainly on the magnitude of an increase in symptoms. ${ }^{4}$ All guidelines for clinical practice recommend that the classification of the stages of heart failure should be applied according to the NYHA (TABLE 1).

The rest ECG and the chest X-ray should constitute essential components of initial diagnostics of heart failure (FIGURE 1). A helpful test, which in the case of correct results virtually enables ruling out heart failure, is the assessment of the B-type natriuretic peptide (BNP, NT-proBNP) level. ${ }^{1,4}$ This test could be particularly useful in primary health care and in hospital emergency units. Other laboratory tests, which are used in initial assessment of a patient being diagnosed for chronic heart failure, include complete blood count, levels of electrolytes, creatinine, glucose, liver enzymes, thyroid hormones in serum and a routine urine analysis. ${ }^{1,4}$

Echocardiography is a method of choice to confirm systolic or diastolic heart dysfunction. 
FIGURE 1 Algorithm

for the diagnosis of heart failure. Guidelines of the European Society of Cardiology for the diagnosis and treatment of Chronic Heart Failure (according to: Kardiol Pol. 2005; 63: 515, adapted)

* full blood count, plasma levels of electrolytes, creatinine, glucose, hepatic enzymes, thyroid hormones and routine urine analysis

** Sometimes this test should be used during earlier diagnostic stage (e.g. diagnosis of heart failure with preserved left ventricular ejection fraction).

Abbreviations: LV - left ventricular, MR - magnetic resonance

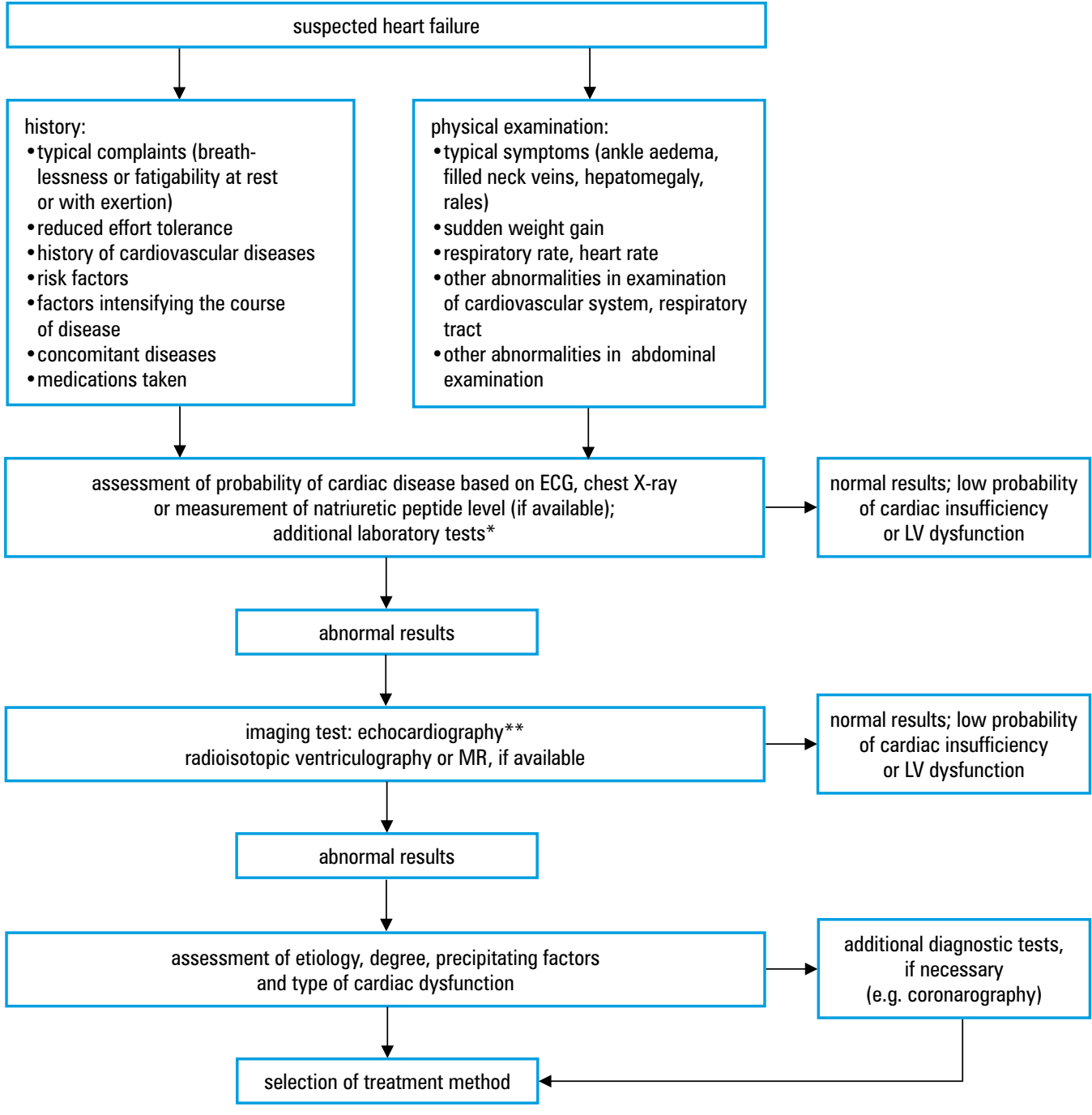

Confirmation of that dysfunction by echocardiography or other imaging methods (cardiac magnetic resonance, radioisotope tests) is necessary to diagnose HF. ${ }^{1-4}$

Invasive tests (coronary angiography, right heart catheterization with hemodynamic measurements, endomyocardial biopsy) are not routine tests essential for diagnosis of chronic HF. ${ }^{1}$ However, in selected clinical situations, they may be helpful in determining a cause of the disease and introducing appropriate therapy. ${ }^{1}$

Treatment algorithm in CHF Treatment in heart failure aims at: preventing the progress of pathophysiological lesions in the cardiovascular system associated with neurohormone activation, decreasing the intensity of complaints, improving the quality of life and increasing survival rates.

Therapy in patients with CHF due to systolic dysfunction involves non-pharmacological measures (guidelines for lifestyle), drug therapy, implanting cardioverter-defibrillators and(or) resynchronization device, mechanical support and surgical treatment. The proposed guidelines for graded non-pharmacological, pharmacological and surgical treatment for $\mathrm{CHF}$ are presented in FIGURE 2.
Pharmacotherapy Angiotensin-converting enzyme inhibitors (ACEI) ACEI treatment is a cornerstone of heart failure therapy in order to reduce morbidity and mortality. The ACEI therapy should be initiated from class I according to the NYHA. There are several studies related to the role of ACEI in patients with symptomatic chronic heart failure (Cooperative North Scandinavian Enalapril Survival Study, Vasodilator-Heart Failure Trial II and Studies of Left Ventricular Dysfunction - with enalapril) and in patients after myocardial infarction (Survival and Ventricular Enlargement trial - with captopril, Acute Infarction Ramipril Efficacy Study - with ramipril, Trandolapril Cardiac Evaluation Study - with trandolapril). Based on those studies, it has been found that convertase inhibitors reduce mortality risk in $\mathrm{CHF}$ by $16 \%{ }^{1}$

Thus the following recommendations have been developed. ${ }^{1}$

Class I, level A. ACEI should be used in all patients with reduced left ventricular ejection fraction (LVEF), on condition that there are no contraindications and tolerance is good.

The role of appropriate dosing ACEI, particularly before starting a therapy with other groups of medications, is being emphasized. The Assessment of Treatment with Lisinopril and Survival 
TABLE 2 Effectiveness of ARB in comparison with ACEl in large randomized controlled clinical trials with hard outcomes

\begin{tabular}{|c|c|c|c|c|c|}
\hline & ELITE I/II & OPTIMAAL & VALIANT & Val-HeFT & CHARM \\
\hline Patients (number) & $\begin{array}{r}\text { NYHA II-IV } \\
722 / 3152\end{array}$ & $\begin{array}{c}\mathrm{Ml} / \mathrm{HF} \\
5477\end{array}$ & $\begin{array}{l}\mathrm{Ml} / \mathrm{HF} \\
14808\end{array}$ & $\begin{array}{l}\text { NYHA II-IV } \\
5010\end{array}$ & $\begin{array}{l}\text { NYHA II-IV } \\
2548\end{array}$ \\
\hline Study design (tested agents) & $\begin{array}{l}\text { losartan vs. } \\
\text { captopril }\end{array}$ & $\begin{array}{l}\text { losartan vs. } \\
\text { captopril }\end{array}$ & $\begin{array}{l}\text { valsartan vs. } \\
\text { captopril vs. both } \\
\text { agents }\end{array}$ & valsartan vs. ACEI & $\begin{array}{l}\text { candesartan vs. } \\
\text { ACEl vs. both } \\
\text { agents }\end{array}$ \\
\hline$\beta$-adrenolytics & $16 \% / 23 \%$ & $79 \%$ & $70 \%$ & $35 \%$ & $55 \%$ \\
\hline Cardiovascular mortality & no difference & favors captopril & no difference & no difference & $\begin{array}{l}\text { favors candesartan, } \\
\text { favors both } \\
\text { agents }\end{array}$ \\
\hline Hospitalizations due to CHF & no difference & favors captopril & favors both agents & favors both agents & favors both agents \\
\hline
\end{tabular}

Abbreviations: ACEI - angiotensin-converting enzyme inhibitors, ARB - angiotensin receptor blockers, CHARM - Candesartan in Heart Failure: Assessment of Reduction in Mortality and Morbidity Programme, CHF - chronic heart failure, ELITE I/II - Losartan Heart Failure Survival Study, HF - heart failure, OPTIMAAL - Optimal Trial in Myocardial Infarction with Angiotensin Il Antagonist Losartan Study, MI - acute myocardial infarction, VALIANT - Valsartan in Acute Myocardial Infarction Study, Val-HeFT - Valsartan Heart Failure Trial

study showed that although treatment with large doses of lisinopril failed to reduce total mortality as compared to small doses of the medication, it reduced the frequency of hospitalization due to exacerbated heart failure. ${ }^{1,5}$ Therefore, the ACEI therapy should not be limited only to achieving symptomatic improvement. However, if it is appropriate to use other medications (particularly $\beta$-adrenolytics), the ACEI treatment should be intensified (up to a target dose) only after the introduction of such medications.

Class I, level A. Doses of ACEI should be gradually increased to achieve the dosage of effectiveness showed in large controlled clinical trials.

The European guidelines provide a list of ACEI, whose effectiveness has been shown in large controlled clinical trials on HF or left ventricular dysfunction. These medications, whose impact on mortality/frequency of hospitalization in $\mathrm{CHF}$ is documented, include: ${ }^{1}$

1) captopril (target dose: $25-50 \mathrm{mg} 3$ times daily)

2) enalapril (target dose: $10 \mathrm{mg}$ twice daily)

3) lisinopril (target dose: 5-20 mg twice daily)

4) ramipril (target dose: $2.5-5 \mathrm{mg}$ twice daily)

5) trandolapril (target dose: $4 \mathrm{mg}$ once daily)

Comparison between the European and American and Canadian guidelines The guidelines are fully unanimous that ACEI constitute a basis for heart failure treatment. The Canadian guidelines, likewise the European ones, list particular medications of that group and their dosage whose effectiveness has been showed in large randomized controlled clinical trials with hard outcomes. The Canadian guidelines clearly prefer and recommend precisely those medications (class I, level A). Slight differences pertain among others to the recommended dosage of lisinopril once daily. The ACC/AHA guidelines provide the wider spectrum of medications used in CHF treatment. They also take fosinopril, perindopril and chinapril into consideration. ${ }^{2}$ The HFSA guidelines do not specify medications or their dosage. $\boldsymbol{\beta}$-adrenolytics $\beta$-adrenolytic therapy should be started in CHF patients in NYHA class II, or in patients after myocardial infarction from class I.

Clinical trials involving $\beta$-adrenolytics in $\mathrm{CHF}$ do not show the class effect. Studies, like the Heart failure Bisoprolol Study II (CIBIS II; with bisoprolol), the Metoprolol CR/XL Randomized Intervention Trial in Congestive Heart Failure (with extended release metoprolol succinate), the Carvedilol Prospective Randomized Cumulative Survival Trial (with carvedilol) and the Study of Effects of Nebivolol Intervention on Outcomes and Rehospitalisation in Seniors with heart failure (with nebivolol) showed effectiveness in reducing morbidity and mortality in CHF patients. ${ }^{1}$ On the other hand, trials involving bucindolol (Beta-Blocker Evaluation of Survival Trial) and short-acting metoprolol (Metoprolol in Dilated Cardiomyopathy Study) did not show beneficial effects of those medications. ${ }^{1,2}$

Thus the following recommendation has been framed. ${ }^{1}$

Class I, level A. $\beta$-adrenolytics (bisoprolol, carvedilol, extended release metoprolol succinate and nebivolol) should be used, if no contraindications, in all patients with stable heart failure (in NYHA classes II-IV).

Observations have shown that higher doses of $\beta$-adrenolytics offer greater clinical benefits. In the Heart failure Bisoprolol Study I study, a $5 \mathrm{mg}$ bisoprolol dose was used and slight improvement, which was not statistically significant, was achieved. Only after the administration of a $10 \mathrm{mg}$ bisoprolol dose in the CIBIS II study, a significant reduction in mortality was showed. However, it should be remembered that reaching the target dose ought to be slow and individualized, and depended on a patient's response. Treatment should be started in patients, who do not display symptoms of hypervolemia, or such symptoms are barely detectabable. Usually, the administration is initiated from quite small doses, which are then gradually increased.1,2,4 
FIGURE 2 Stages

of non-pharmacological, pharmacological and surgical treatment for heart failure.

Abbreviations: ACC American College of Cardiology, ACEI angiotensin-converting enzyme inhibitors, AHA American Heart Association, ARB - angiotensin receptor blockers, $\mathrm{BP}$ blood pressure, ESC European Society of Cardiology

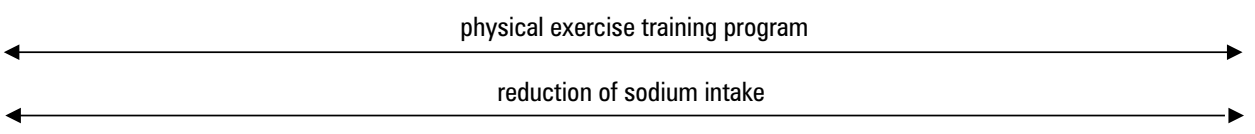

Comparison between the European and American and Canadian guidelines In respect of use of $\beta$-adrenolytics in $\mathrm{CHF}$, the recommendations are generally unanimous. The Canadian guidelines extend only a scope of use of those medications to all patients with $\mathrm{LVEF} \leq 40 \%$, irrespective of the NYHA class. ${ }^{4}$ The ACC/AHA and the CCS guidelines recommend bisoprolol, carvedilol, extended release metoprolol succinate (the European guidelines also recommend nebivolol).

Aldosterone receptor antagonists The Randomized Aldactone Evaluation Study showed that in patients with advanced CHF (NYHA III-IV) and LVEF $\leq 35 \%$, adding spironolactone to the ACEI treatment with a diuretic and digoxin (most patients) significantly reduces mortality. ${ }^{2,4}$ Only $10 \%$ of patients involved in that study were administered $\beta$-adrenolytics. ${ }^{2,4}$

The following recommendation has been issued. ${ }^{1}$

Class I, level B. It is recommended to use aldosterone receptor antagonists along with ACEI, $\beta$-adrenolytics and diuretics in patients with advanced CHF (NYHA III-IV) due to left ventricular systolic dysfunction (LVSD), because it reduces mortality and morbidity in those patients.

The treatment mentioned above should be provided only in patients, in whom it is possible to monitor closely the renal function and the serum potassium level. The medications recommended in the guidelines are spironolactone and eplerenon in a target dosage of $50 \mathrm{mg}$ daily. ${ }^{1,2,4}$ A large recommended target dose of spironolactone is worth mentioning, in particular with other medications having an effect on the renin-angiotensin system (RAS). It is notable that in the Randomized Aldactone Evaluation Study, a mean daily dose of spironolactone was $26 \mathrm{mg} .{ }^{6}$ The researchers emphasized in their commentary that severe hyperkalemia had most frequently occurred in patients receiving $50 \mathrm{mg}$ of spironolactone daily. ${ }^{6}$

Comparison between the European and American and Canadian guidelines As opposed to the European, Canadian and ACC/AHA guidelines, which unanimously recommend the administration of aldosterone receptor antagonists in class I recommendations with the B level of evidence, the HFSA recommendations assign class I and the A level of evidence to that indication. It is associated with the fact that the HFSA, in their assessment of the level of evidence, took the results of the Eplerenone Post-Acute Myocardial Infarction Heart Failure Efficacy and Survival Study into account. ${ }^{3}$ This study showed favorable effects of eplerenone in patients early after acute myocardial infarction, with low LVEF $(\leq 40 \%)$ and heart failure or diabetes. ${ }^{3}$ The Canadian guidelines additionally recommend the use of those medications only in patients with LVEF below $30 \%{ }^{4}$

Angiotensin receptor blockers (ARB) It is well known that in patients with symptomatic $\mathrm{CHF}$ despite the ACEI treatment, the level of angiotensin II increases. Responsible for this phenomenon are additional enzymatic path ways (e.g. associated with chymase), where angiotensin II is produced. It provides theoretical rationale for the use of ARB, also combined with ACEI. The results of clinical studies that compared the effectiveness of ACEI and ARB were, however, ambiguous (TABLE 2).

It was a basis for the following recommendations. ${ }^{1}$

Class I, level B. In patients with heart failure symptoms, ARB may be used instead of ACEI, in case of intolerance. Such treatment reduces mortality and morbidity.

Class IIa, level B. ARB and ACEI seem to be equally effective in the reduction of mortality and morbidity.

Class I, level B. Impact of medications from both groups on mortality in the course of acute myocardial infarction with symptoms of heart failure or left ventricular dysfunction is similar or the same.

Class IIa, level B. ARB and ACEI combined therapy may be considered, if, despite the use of one medication, clinical symptoms persist. This therapy reduces mortality.

Class I, level A. Such treatment also reduces the risk of hospitalization due to heart failure. 
TABLE 3 Comparison of guidelines for use of ARB in CHF in different guidelines

\begin{tabular}{lllllllll} 
& ESC & \multicolumn{3}{c}{ ACC/AHA } & CCS & & HFSA \\
& Class & Level & Class & Level & Class & Level & Class & Level \\
\hline ACEl intolerance & I & B & I & A & I & A & I & A \\
\hline Along with ACEI & - & - & llb & B & I & A & Ila & A \\
\hline Mortality reduction $^{*}$ & Ila & B & - & - & - & - & - & - \\
\hline Hospitalization frequency reduction $^{*}$ & I & A & - & - & - & - & - & - \\
\hline
\end{tabular}

${ }^{*}$ Only ESC guidelines distinguish the influence of ARB on different outcomes.

Abbreviations: ACC - American College of Cardiology, AHA - American Heart Association, CCS - Canadian Cardiovascular Society, HFSA - Heart Failure Society of America, ESC - European Society of Cardiology, others - see TABLE 2

Comparison between the European and American and Canadian guidelines The European and Canadian guidelines list ACEI whose effectiveness has been shown in large controlled clinical trials on heart failure or left ventricular dysfunction. Medications of this group, which in this indication has documented an impact on mortality/hospitalization frequency comprise as follows: ${ }^{1,4}$

1) candesartan (target dose: $32 \mathrm{mg}$ )

2) valsartan (target dose: $320 \mathrm{mg}$ ).

The ACC/AHA guidelines additionally mention losartan as a medication used in cardiac insufficiency with left ventricular systolic dysfunction (target dose: $50-100 \mathrm{mg}$ ). ${ }^{2}$

The existing divergences in the results of clinical trials on heart failure using ARB (TABLE 2) resulted the guidelines for use of this group of medications, proposed by the Societies (TABLE 3).

In case of intolerance of ACEI, the existing differences in guidelines of different Societies are few and have no practical significance. Significant divergences appear, however, with the use of ARB in patients with maintaining symptoms of heart failure, despite appropriate treatment with ACEI and $\beta$-adrenolytics. In such cases, the ESC guidelines themselves are not coherent in respect of combined administration of ACEI and ARB and the moment of introduction of such therapy in a symptomatic patient. In chart no 4 in the original text of the guidelines (FIGURE 3 in this paper), adding ARB is recommended in patients who, despite the use of ACEI and $\beta$-adrenolytics remain in NYHA functional class III. ${ }^{1}$ In Table 22 in the original text of the guidelines, such combination is acceptable already in patients in NYHA class II. ${ }^{1}$ This ambiguity is probably associated with the results of 2 large clinical trials which showed the effectiveness of such combination: the Valsartan Heart Failure Trial (Val-HeFT) and the Candesartan in Heart Failure - Added Trial (CHARM-Added trial). ${ }^{7,8}$ Both trials involved patients in NYHA functional class II-IV. In the Val-HeFT trial the majority were the patients included in NYHA functional class II (62\%), and in the CHARM-Added trial, patients in NYHA class III (73\%). 7,8

Furthermore, in the case of combined use of ACEI and ARB in CHF, the European guidelines introduce a new approach: determination of the recommendation class and recommendation level depending on the anticipated clinical effect. ${ }^{1}$ It is closely related to the results of clinical trials. Since a reduction in the hospitalization frequency in CHF, as a result of ACEI + ARB combined treatment was observed in two large clinical trials (CHARM-Added and Val-HeFT) $)^{7,8}$, this indication was assigned class I and the A level of recommendations by the ESC ${ }^{1}$. And because a favorable impact on mortality in $\mathrm{CHF}$ was shown only in the CHARM-Added trial ${ }^{7,8}$, this indication was assigned a lower level of recommendations by the ESC (IIa B) ${ }^{1}$. The Canadian guidelines generally grant the combination of ACEI and ARB in CHF a high level of recommendations (I A). ${ }^{4}$ The commentary states it pertains to a reduction in the hospitalization frequency. ${ }^{4}$ The American guidelines also do not diversify outcomes and anticipated clinical effects. The HFSA guidelines do not generally differ from the $\mathrm{Eu}$ ropean and Canadian guidelines, and the analyzed combination has a recommendation level of IIa A. ${ }^{3}$ The ACC/AHA guidelines significantly differe from the others and limit the clinical significance of ACEI + ARB combination (recommendation level IIb B). ${ }^{2}$

The described differences show how diversified could be interpretations of results of large clinical trials carried out according to the evidence-based medicine (EBM) rules.

\section{Cardiac glycosides}

Class I, level B. Cardiac glycosides are recommended in atrial fibrillation and symptomatic heart failure of any degree.

Class IIa, level A. Digoxin may reduce the frequency of hospitalization due to CHF in patients with preserved sinus rhythm and decreased LVEF.

The recent re-analysis of the DIG trial has shown that in subjects with low plasma digoxin levels $(0.5-0.9 \mathrm{ng} / \mathrm{ml})$ not only a reduction in the frequency of hospitalization, but also a significant reduction in mortality can be observed. ${ }^{9}$ The administered digoxin doses should probably be reduced to $\leq 0.125 \mathrm{mg}$ daily. ${ }^{9}$ 
of symptomatic heart failure due to left ventricular systolic dysfunction (according to: Kardiol Pol. 2005; 63: 535, adapted)

Note: In case of ACEI, ARB and $\beta$-adrenolytics, efforts should be made to achieve recommended doses, i.e. the doses, whose effectiveness has been demonstrated by large randomized controlled clinical trials with hard outcomes.

*In Table 22 of the original text of guidelines, such combination is admissible already in NYHA II patients (Kardiol Pol. 2005; 63: 534). **Low doses of digoxin $0.125 \mathrm{mg}$ daily should be considered.

Abbreviations: $\mathrm{Ml}$ - myocardial infarction, others - see Figure 2
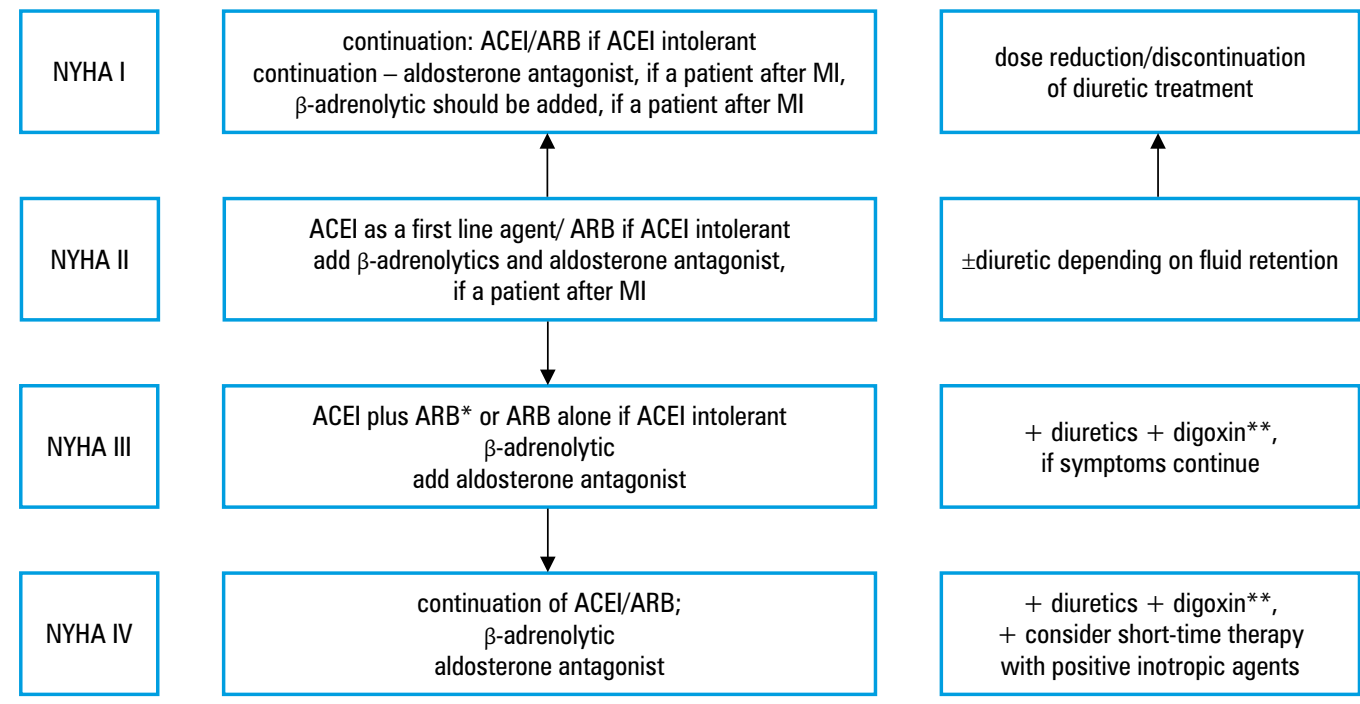

Comparison between the European and American and Canadian guidelines The existing divergences between guidelines concern the use of digoxin in patients with preserved sinus rhythm. The HFSA recommendations are similar to the European ones. ${ }^{3}$ The ACC/AHA guidelines are similar, and differ only in the assigned lower level of evidence (B). ${ }^{2}$ The Canadian guidelines acknowledge recommendations class I with the A level of clinical evidence for this indication. ${ }^{4}$ The existing differences are probably associated with the fact that most studies documenting the effectiveness of digoxin come from the period, when other medications (particularly $\beta$-adrenolytics and ARB) were used less frequently. They also signify that in drawing up the guidelines for clinical practice "expert opinions" may be substantial in the assessment of significance of scientific evidence. ${ }^{10}$

\section{Hydralazine - isosorbide dinitrate}

Class IIa, level B. In case of intolerance of ACEI and $A R B$ and renal insufficiency, an attempt to treat with hydralazine/nitrates may be made in order to reduce mortality and morbidity and to improve the quality of life.

Comparison between the European and American and Canadian guidelines The ACC/AHA and Canadian guidelines assign for this indication a lower recommendation of class IIb and the level of evidence B or C. ${ }^{2,4}$ However, it seems that the available scientific evidence does not show that in patients not tolerating ACEI/ARB, particularly in renal insufficiency, the combination of hydralazine and nitrates may bring beneficial clinical effects. ${ }^{10}$

\section{Diuretics}

Class I, level A. Diuretics are a basis for treatment mitigating the symptoms in case of hypervolemia manifested by pulmonary congestion or peripheral edema. ${ }^{1}$ The use of those medications reduces heart failure symptoms.

Rules for diuretic use Diuretics, as compared to other agents used in CHF, cause the quickest symptomatic improvement in patients with signs of pulmonary and(or) systemic congestion. ${ }^{2}$

Appropriate use of diuretics influences the effectiveness of therapy with other medications in CHF. Too small doses may cause fluid retention, reduce a response to ACEI and increase the risk of treatment with $\beta$-adrenolytics. In turn, too large doses of diuretics may increase the risk of hypotonia, when taking ACEI, and renal insufficiency, when taking ACEI and ARB. ${ }^{2}$ The objective of treatment is a reduction in congestion and body weight, usually of $0.5-1 \mathrm{~kg}$ daily. ${ }^{2}$ Normally, loop diuretics are recommended as first-line agents. ${ }^{2,4}$ Furosemide is used most frequently. Many patients respond well to a newer loop diuretic, i.e. torsemide, which has better biological availability and longer effects. ${ }^{2}$ Thiazide diuretics (when glomerular filtration rate $>30-40 \mathrm{ml} / \mathrm{min}$ ), potassium-saving diuretics or their combinations may also be used.

Potassium level should be preserved definitely above $4 \mathrm{mmol} / \mathrm{l}$. $^{4,9,11}$

After achieving clinical improvement, it is appropriate to use a diuretic chronically in the smallest dose essential to maintain a patient in a stable condition. ${ }^{4}$ Caution is necessary in chronic use of diuretics, because to date no results of larger, randomized clinical trials assessing the outcomes of treatment with this group of medications are available. Diuretics may potentially intensify neurohormone activation, which is increased in CHF. The analysis of patients involved in the DIG trial, which was made using the propensity score in order to reduce the influence of disturbing factors, showed that the CHF patients treated with a diuretic manifested higher total mortality $(29 \%$ 
vs. $21 \%$, HR $1.31, \mathrm{p}=0.002)$ and higher mortality due to HF ( $9 \%$ vs. $6 \%$ HR $1.36, p=0.056) .{ }^{11}$ These differences were found irrespective of etiology of heart failure, the NYHA class, ejection fraction and ACEI treatment. ${ }^{11}$ However, it should be noted that such retrospective analyses have their limitations, because in everyday practice, patients requiring diuretics or their large doses most frequently demonstrate more advanced stages of CHF. It may have an effect on higher mortality observed in that group.

Selection of a pharmacological treatment method Based on the analyzed European guidelines, we could present a detailed algorithm of pharmacotherapy for symptomatic HF caused by left ventricular systolic dysfunction (FIGURE 3 ).

Selecting a proper dose of medications mentioned in FIGURE 3, it should be noted that:

1) In case of ACEI and ARB, the dose should be increased gradually up to the recommended one, i.e. the dose used in large, randomized, controlled clinical trials with hard outcomes. The intended dose must be tolerated by a patient.

2) In patients, in whom the hypervolemia signs have been overcome (mainly with ACEI and diuretics), $\beta$-adrenolytic therapy should be introduced. The dose ought to be increased gradually up to the target one, determined based on large, controlled clinical trials.

3) Diuretic dosage should be adjusted individually depending on a patient's clinical condition based on intensity of symptoms.

The management of a CHF patient according to the presented algorithms should be based on a patient's thorough history concerning intensification of symptoms and toleration of treatment. It is still an activity involving elements of the art of medicine, i.e. subjective assessment made by an attending physician and his/her clinical experience. Taking an appropriate and in-depth history of a patient during control visits plays a considerable role in the optimization of $\mathrm{CHF}$ therapy. The role of appropriately selected medical teams in chronic care of $\mathrm{CHF}$ patients should also be emphasized. Apart from a cardiologist, such teams need to include a general practitioner, a trained nurse, a clinical psychologist and a physiotherapist. The role of a nurse in this system of multidisciplinary care is crucial. The Argentinean DIAL study showed that in $\mathrm{CHF}$ patients randomized to a group, where a routine care was expanded by telephone control of clinical condition, training and modifications in treatment of dehydration carried out by a trained nurse, a $29 \%$ reduction in need for hospitalization due to heart failure $(\mathrm{p}<0.005)$ was observed. ${ }^{12}$ In their findings, Ezekowitz et al. emphasized the role of a cardiologist in chronic care of a CHF patient. In patients who, after hospital discharge, were cared for by a general practitioner and a cardiologist, mortality was lower than in patients attended only by a general practitioner
(17\% vs. $28 \%$, p <0.001). ${ }^{13}$ Similar benefits from the program of multidisciplinary outpatient clinic treatment after hospital discharge were shown by Wierzchowiecki et al. also in the Polish population. ${ }^{14}$ The experience of the attending team and frequent control visits in an outpatient clinic (14 days after hospital discharge and at the $3 \mathrm{rd}$, 6 th, 9th and 12th months) are thought to have been significant in achieving such positive results. Attention should also be paid to the fact that in case of difficult patients (quick, treatment-resistant progression of heart failure, suspected inflammatory etiology), particularly during the first phase of treatment, a relevant role may be played by a consultation provided by a cardiologist specializing in $\mathrm{CHF}$ treatment, in order to expand the diagnostics and tailor therapy to the patient needs.

Lack of satisfactory methods of CHF patient treatment objective assessment is one of the reasons for the limited introduction of the presented guidelines to everyday medical practice. ${ }^{5,15}$ The recent Euro Heart Survey on heart failure, performed by the ESC, demonstrated that only $78 \%$ of patients with CHF and LVSD were treated with ACEI. ${ }^{5}$ Only $46 \%$ of patients were receiving $\beta$-adrenolytics, and $29 \%$ aldosterone antagonist. ${ }^{5}$ In that European study involving 10,701 patients, the doses of administered medications were significantly lower than those used in large controlled clinical trials. ${ }^{5}$ Only half of patients who met the inclusion criteria in the SOLVD study, were receiving a target dose of ACEI, and only $10 \%$ of patients, who met the criteria for enrollment in the MERIT-HF study, were receiving $\beta$-adrenolytics in a recommended dose. ${ }^{5}$

An attempt to make the chronic treatment of $\mathrm{CHF}$ patients more objective is the inclusion of biomarkers, especially BNP/NT-proBNP, in the therapeutic scheme. ${ }^{16}$ The recently published, multicenter, randomized STARS-BNP trial confirmed that the $\mathrm{CHF}$ treatment with BNP determination may provide greater benefits than the therapy based only on clinical symptoms. ${ }^{15}$ In a group of patients, where BNP measurements were additionally used to select the intensity of pharmacological treatment, it was found that the use of larger doses of basic medications in $\mathrm{CHF}$ therapy (up to $106 \%$ of doses recommended for ACEI and $77 \%$ for $\beta$-adrenolytics) is translated into less frequent occurence of a combined outcome, i.e. deaths and hospitalizations associated with $\mathrm{CHF}$ (24\% in the BNP group and 52\% in the control group; $\mathrm{p}<0.001) .{ }^{5}$ These results are promising, but have to be confirmed by studies involving more numerous groups of patients and based on hard outcomes.

Interventional treatment Cardiac resynchronization therapy (CRT) by means of biventricular stimulation or biventricular stimulation combined with cardioverter-defibrillator.

Class I. Introduction of CRT may be considered in patients with lowered LVEF ( $\leq 35 \%)$, left 
* during the research of CRT, different left ventricular (LV) widening criteria were applied: LV end diastolic dimension $>55 \mathrm{~mm}$, LV end systolic dimension $>30 \mathrm{~mm} / \mathrm{m}^{2}$, LV end systolic dimension $>30 \mathrm{~mm} / \mathrm{m}$ of height ventricular hypertrophy*, sinus rhythm and synchronization disorders (QRS width $\geq 120 \mathrm{~ms}$ ), with persisting despite optimum pharmacological treatment CHF symptoms (III-IV NYHA class). 1,17

Receiving CRT-P reduces:

1) clinical symptoms (class $I$, level $A)^{1,17}$

2) frequency of hospitalization (class $I$, level $A)^{1,17}$

3) mortality (class I, level A). ${ }^{17}$

The use of CRT with cardioverter defibrillator is an accepted alternative method of treatment in such patients, on condition that the expected time of their survival in a good functional condition exceeds 1 year (class I, level B). ${ }^{17}$

The metaanalysis of studies concerning resynchronizing therapy (Multisite Stimulation in Cardiomyopathy study; Multicenter InSync Randomized Clinical Evaluation Trial; Multisite Stimulation in Cardiomyopathy Study; Comparison of Medical Therapy, Pacing, and Defibrillation in Heart Failure Trial; Cardiac Resynchronization-Heart Failure Trial) confirmed a favorable influence of the treatment on advanced heart failure outcomes. ${ }^{18}$ In comparison with optimal pharmacotherapy, after administering resynchronization therapy, a reduction was found in total mortality by $29 \%$ (odds ratio [OR] $0.71,95 \%$ CI $0.57-0.88$ ) and in mortality associated with $\mathrm{CHF}$ progression by $38 \%$ (OR, 0.62, 95\% CI 0.45-0.84)..$^{15}$

The role of echocardiography in qualifying patients for resynchronization therapy is currently being investigated, because clinical and ECG criteria recommended by the guidelines are not always sufficient. However, recently published results of the PROSPECT study failed to show that currently used ECG manifestations of LV systolic dyssynchrony were useful in qualifying patients for resynchronization therapy. ${ }^{19}$

Comparison between the European and American and Canadian guidelines The American, Canadian and European guidelines (for cardiac stimulation and resynchronization) provided the detailed value of LVEF ( $\leq 35 \%)$, for which the introduction of resynchronization therapy is recommended and assign this form of CHF treatment with the similar class of recommendation (only the HFSA with class IIa). ${ }^{1-4,17}$ They, however, do not diversify the anticipated clinical effects and recognize the A level of evidence for this indication. It seems to be justified, especially after drawing the conclusions from the mentioned above metaanalysis. ${ }^{18}$

\section{Implanted cardioverter-defibrillators (ICD)}

\section{Secondary prevention}

Class I, level A (to reduce mortality). The implantation of ICD is recommended in patients who survived circulatory arrest caused by ventricular fibrillation, and in patients with poorly hemodynamically tolerated ventricular tachycardia (VT) who undergo optimum pharmacotherapy and are anticipated to survive in a good functional condition for over one year. ${ }^{1,20}$

\section{Primary prevention}

Class I, level A (to reduce mortality). In selected patients with LVEF $\leq 30-40 \%$ who are of II or III NYHA class, on condition that they already undergo optimum pharmacotherapy and are anticipated to survive in a good functional condition for over one year, however, not earlier than 40 days after myocardial infarction. ${ }^{1,20}$

Class I, level B (to reduce mortality). The implantation of ICD is recommended in patients with heart disease of etiology other than ischemic, with LVEF $\leq 30-35 \%$, being of II or III NYHA class, undergoing optimum chronic pharmacological treatment and with anticipated survival in a good functional condition for over 1 year. ${ }^{20}$

Class IIa, level B (to reduce mortality). The implantation of ICD is justified in patients with impaired $L V$ function due to MI from at least 40 days before, with LVEF $\leq 30-35 \%$, being of I NYHA class, who undergo optimum pharmacotherapy and are anticipated to survive in a good functional condition for over one year. ${ }^{20}$

Class IIb, level B (to reduce mortality). The implantation of ICD is justified in patients with heart disease of etiology other than ischemic, with LVEF $\leq 30-35 \%$, being of I NYHA class, who undergo optimum pharmacotherapy and are anticipated to survive in a good functional condition for over one year. ${ }^{20}$

Comparison between the European and American and Canadian guidelines In primary prevention of sudden cardiac death (SCD), the American and Canadian guidelines consider patients with lower LVEF in class I recommendations (<30\%). ${ }^{2-4}$ The European guidelines for heart failure and the HFSA ones do not diversify left ventricular systolic dysfunction in respect of etiology. ${ }^{1,3}$ The European recommendations for the prevention of SCD and the ACC/AHA and the CCS for the use of ICD in patients with systolic dysfunction of etiology other than ischemic, are of a lower degree (ESC - I B or IIb B, ACC/AHA - I B; CCS - IIa B or IIb $B$ in patients with LVEF 31-35\%). 2,4,20

Heart revascularization and related surgeries $\mathrm{All}$ guidelines for treatment for $\mathrm{CHF}$ concur that in case of patients with symptoms of angina pectoris it is appropriate to consider surgical or percutaneous heart revascularization (class I or IIa and levels of recommendation A or B).

Revascularization in order to improve myocar dial contractility or achieve a reduction in $\mathrm{CHF}$ symptoms is not recommended in case of no symptoms of angina pectoris (even in the case of hibernated myocardium). ${ }^{1}$

Explicitly recommended surgery in CHF patients is excision of the left ventricular aneurism. It is recommended in patients with a large, sectioned off aneurism and signs of heart fail ure (class I, level C). ${ }^{1}$ Cardiac transplantation is also a recommended method of treatment of final stage heart failure, providing the patients 
TABLE 4 Recommendations for treatment of patients with heart failure and normal LVEF (according to AHA/ACC and CCS) ${ }^{2,4}$

\begin{tabular}{|c|c|c|}
\hline Recommendation & Class & Level \\
\hline $\begin{array}{l}\text { Control of systolic and diastolic pressure, in accordance } \\
\text { with current recommendations. }\end{array}$ & I & A \\
\hline $\begin{array}{l}\text { Control of ventricular frequency in patients with atrial } \\
\text { fibrillation. }\end{array}$ & I & C \\
\hline $\begin{array}{l}\text { Diuretics to control pulmonary congestion and peripheral } \\
\text { edema. }\end{array}$ & I & C \\
\hline $\begin{array}{l}\text { Surgical revascularization of myocardium in symptomatic } \\
\text { patients with coronary disease or documented significant } \\
\text { cardiac ischemia }\end{array}$ & Ila & C \\
\hline $\begin{array}{l}\text { Restoration and preservation of sinus rhythm in patients } \\
\text { with atrial fibrillation to reduce symptoms. }\end{array}$ & llb & C \\
\hline $\begin{array}{l}\text { Use of } \beta \text {-adrenolytics, ACEI, ARB or calcium antagonists in } \\
\text { hypertensive patients may be effective in heart failure } \\
\text { symptoms reduction. }\end{array}$ & llb & C \\
\hline $\begin{array}{l}\text { Use of digoxin to reduce heart failure symptoms has } \\
\text { no sufficient justification. }\end{array}$ & llb & C \\
\hline $\begin{array}{l}\text { In all patients, use of ACEI and } \beta \text {-adrenolytics should be } \\
\text { considered. }{ }^{4}\end{array}$ & Ila & B \\
\hline
\end{tabular}

Abbreviations: LVEF - left ventricular ejection fraction, others - see TABLE 2 and 3

are properly qualified to the surgery (class $I$, level C). ${ }^{1}$

Surgical corrections of serious functional mitral regurgitation in patients with advanced LVSD in order to reduce the CHF symptoms are promising, although they still have a relatively low level of recommendation (class IIb, level C). ${ }^{1}$

Selected other recommendations to the scheme of treatment resulting from the guidelines ${ }^{1-4}$

1) exercise in a stable period and the routine conduct of training programs (class I, level C)

2) control of body weight by a patient in respect of possible rapid weight gain (class I, level C)

3) in case of fluid retention: reduction in salt supply (usually $2-3 \mathrm{~g}$ daily), reduction in fluid supply (usually 1.5-2 1 daily) (class I, level C)

4) anticoagulant therapy:

a) with concomitant atrial fibrillation (class I, level $A)^{1}$

b) history of thromboembolism (class I, level $A)^{1}$

c) mobile left ventricular thrombus (class I, level $A)^{1}$

d) in secondary prevention of coronary heart disease it is recommended to administer acetylsalicylic acid or oral anticoagulants (class IIa, level C) ${ }^{1}$

e) chronic use of the combination of antiplatelet agents and anticoagulants is not recommended, except for individual cases (class III, level $A)^{4}$

5) avoidance of the use of acetylsalicylic acid in patients who require frequent hospitalization due to heart failure exacerbation (class IIb, level B) ${ }^{1}$

6) avoidance of non-steroid anti-inflammatory agents (class I, level C) ${ }^{1}$
7) avoidance of calcium antagonists (except for amlodipine) (class I, level C) ${ }^{1}$

8) using class I antiarrhythmic agents is contraindicated, because they increase mortality in CHF patients (class III, level B); the medication of choice (except for $\beta$-adrenolytics) is amiodarone, which is effective in controlling most of supraventricular and ventricular arrhythmias (class I, level A)

9) after implanting ICD, it is recommended to use amiodarone, sotalol and(or) other $\beta$-adrenolytics as a method of pharmacological supplementation to ICD therapy to mitigate symptomatic ventricular tachyarrhythmias in HF patients receiving optimal pharmacotherapy (class I, level C) ${ }^{20}$

10) routine use of the combination of ACEI, ARB and spironolactone is not recommended due to an increased risk of hyperkalemia (class III, level C) $)^{2}$

11) influenza vaccination - yearly (class I, level $B)^{21}$, pneumococcal pneumonia vaccination - every 6 years (class I, level C). ${ }^{4}$

Heart failure with preserved left ventricular ejection fraction Heart failure with preserved left ventricular ejection fraction (LVEF $>40 \%$ ) is a vital clinical issue. Approximately $40-50 \%$ of patients with $\mathrm{CHF}$ symptoms have a preserved left ventricular ejection fraction. ${ }^{4}$ Heart failure with preserved left ventricular ejection fraction is not identical with heart failure caused by diastolic dysfunction. ${ }^{1}$ Diagnosis of heart failure with preserved left ventricular ejection fraction does not indicate that characteristics of left ventricular diastolic dysfunction were found. ${ }^{1}$ The latter could be difficult, particularly in patients with atrial fibrillation. ${ }^{1}$

Hints on treatment of heart failure with preserved left ventricular ejection fraction coming from clinical or follow-up studies are scarce. Therefore, in the European guidelines we can find the following statement: "There is no unambiguous evidence demonstrating that patients with initially diastolic heart failure benefit from taking individual medications in any way". ${ }^{1}$ The American and Canadian guidelines are more detailed in their approach to the methods of treatment of CHF patients with preserved LVSF. ${ }^{2,4}$ They are presented in TABLE 4 and they conform with the more general European recommendations.

The paper presents a proposal of the diagnostic and therapeutic scheme in chronic heart failure, worked out based on current guidelines of scientific societies, to be applied in clinical practice. It should be noted that the schemes require periodical updating, because progress in medical knowledge in the field of heart failure is continuous and leads to consistent amendments to the guidelines themselves. 


\section{REFERENCES}

1 Swedberg K, Cleland J, Dargie $\mathrm{H}$, et al. Guidelines for the diagnosis and treatment of chronic heart failure: executive summary (update 2005: The Task Force for the Diagnosis and Treatment of Chronic Heart Failure of the European Society of Cardiology. Eur Heart J. 2005; 26: 1115-1140.

2 Hunt SA; American College of Cardiology; American Heart Association Task Force on Practice Guidelines. ACC/AHA 2005 guideline update for the diagnosis and management of chronic heart failure in the adult. J Am Coll Cardiol 2005;46:e1-e82. Erratum in: JAm Coll Cardiol. 2006; 47 1503-1505.

3 Heart Failure Society Of America. Executive summary: HFSA 2006 Com prehensive Heart Failure Practice Guideline. J Card Fail. 2006; 12: 10-38.

4 Arnold JM, Liu P, Demers C, et al. Canadian Cardiovascular Society Canadian Cardiovascular Society consensus conference recommendations on heart failure 2006: diagnosis and management. Can J Cardiol. 2006 22: 2345 .

5 Lenzen MJ, Boersma $E$, Reimer WS, et al. Under-utilization of evidence-based drug treatment in patients with heart failure is only partially explained by dissimilarity to patients enrolled in landmark trials: a report from the Euro Heart Survey on Heart Failure. Eur Heart J. 2005; 26: 2706-2713

6 Pitt B, Zannad F, Remme W, et al. The Randomized Aldactone Evaluation Study Investigators, The Effect of Spironolactone on Morbidity and Mortality in Patients with Severe Heart Failure. N Engl J Med 1999341: 709-717.

7 Cohn JN, Jay N, Tognoni G, Gianni; the Valsartan Heart Failure Trial Investigators. A randomized trial of the angiotensin-receptor blocker valsartan in chronic heart failure. N Engl J Med. 2001;345: 1667-1675

8 McMurray JJ, Ostergren J, Swedberg K, et al. Effects of candesartan in patients with chronic heart failure and reduced left-ventricular systolic function taking angiotensin-converting-enzyme inhibitors: the CHARM-Added trial. Lancet. 2003; 362(9386):767-771.

9 Ahmed A, Pitt B, Rahimtoola SH, et al. Effects of digoxin at low serum concentrations on mortality and hospitalization in heart failure: $A$ propensity-matched study of the DIG trial. Int J Cardiol. 2008; 123: 138-146.

10 McMurray JJ, Swedberg K. Treatment of chronic heart failure: a comparison between the major guidelines. Eur Heart J. 2006; 27: 1773-1777.

11 Ahmed A, Young JB, Love TE, et al. Heart failure, chronic diuretic use and increase in mortality and hospitalization: an observational study using propensity score methods.. Eur Heart J. 2006; 27: 1431-1439.

12 GESICA Investigators. Randomised trial of telephone intervention in chronic heart failure: DIAL trial. BMJ. 2005; 331: 425.

13 Ezekowitz JA, van Walraven C, McAlister FA, et al. Impact of specialist follow-up in outpatients with congestive heart failure. CMAJ. 2005; 172: $189-194$.

14 Wierzchowiecki M, Poprawski K, Nowicka A, et al. A new programme of multidisciplinary care for patients with heart failure in Poznań: one-year follow-up. Kardiol Pol. 2006; 64: 1063-1070.

15 Jourdain $\mathrm{P}$, Jondeau G, Funck F, et al. Plasma brain natriuretic peptide-guided therapy to improve outcome in heart failure: The STARS-BNP multicenter study. J Am Coll Cardiol 2007; 49: 1733-1739.

16 Gackowski A, Isnard R, Golmard JL, et al. Comparison of echocardiog raphy and plasma B-type natriuretic peptide for monitoring the response to treatment in acute heart failure. Eur Heart J. 2004; 25: 1788-1796.

17 Vardas PE, Auricchio A, Blanc JJ, et al. Guidelines for cardiac pacing and cardiac resynchronization therapy: The Task Force for cardiac pacing and cardiac resynchronization therapy of the European Society of Cardiolo gy. Eur Heart J. 2007; 28: 2256-2295

18 Rivero-Ayerza M, Theuns DA, Garcia-Garcia HM, et al. Effects of cardiac resynchronization therapy on overall mortality and mode of death: a meta-analysis of randomized controlled trials. Eur Heart J. 2006; 27: 2682-2688

19 Cleland JG, Abdellah AT, Khaleva, 0 et al. Clinical trials update from the European Society of Cardiology Congress 2007: 3CPO, ALOFT, PROS PECT and statins for heart failure. Eur J Heart Fail. 2007; 9:1070-1073.

20 Zipes DP, Camm AJ, Borggrefe M, et al. ACC/AHA/ESC 2006 guidelines for management of patients with ventricular arrhythmias and the prevention of sudden cardiac death. Europace. 2006; 8: 746-837.

21 Smith S, Allen J, Blair S, et al. AHA/ACC guidelines for secondary prevention for patients with coronary and other atherosclerotic vascular disease: 2006 update. J Am Coll Cardiol. 2006; 47: 2130-2139.

\section{Trials and studies discussed in the article}

CONSENSUS I (Cooperative North Scandinavian Enalapril Survival Study) V-HeFT II (Vasodilator-Heart Failure Trial II) SOLVD (Studies of Left Ventricular Dysfunction) SAVE (Survival and Ventricular Enlargement trial) AIRE (Acute Infarction Ramipril Efficacy Study) TRACE (Trandolapril Cardiac Evaluation Study)

ATLAS (Assessment of Treatment with Lisinopril and Survival) CIBIS I (Heart failure Bisoprolol Study I) CIBIS II (Heart failure Bisoprolol Study II)

MERIT-HF (Metoprolol CR/XL Randomized Intervention Trial in Congestive Heart Failure)

US CARVEDILOL COPERNICUS (Carvedilol Prospective Randomized Cumulative Survival Trial)

SENIORS (Study of Effects of Nebivolol Intervention on Outcomes and Rehospitalisation in Seniors with heart failure)

BEST (Beta-Blocker Evaluation of Survival Trial)

MDC (Metoprolol in Dilated Cardiomyopathy Study)

RALES (Randomized Aldactone Evaluation Study)

EPHESUS (Eplerenone Post-Acute Myocardial Infarction Heart Failure Efficacy and Survival Study)

ELITE I/II (Losartan Heart Failure Survival Study)

OPTIMAAL (Optimal Trial in Myocardial Infarction with Angiotensin II Antagonist Losartan Study)

VALIANT (Valsartan in Acute Myocardial Infarction Study)

Val-HeFT (Valsartan Heart Failure Trial)

CHARM (The Candesartan in Heart failure: Assessment of Reduction in Mortality and morbidity programme)

CHARM-Added (Candesartan in Heart Failure - Added Trial)

MUSTIC (The Multisite Stimulation in Cardiomyopathy study) MIRACLE (Multicenter InSync Randomized Clinical Evaluation Trial) MUSTIC AF (Multisite Stimulation in Cardiomyopathy Study) COMPANION (Comparison of Medical Therapy, Pacing, and Defibrillation in Heart Failure Trial)

CARE-HF (Cardiac Resynchronization-Heart Failure Trial) 